\title{
A subdiffusion model for granular discharge in a submerged silo
}

Miles L. Morgan, David W. James, Martin Monloubou, and Bjørnar Sandnes

Faculty of Science and Engineering, Swansea University, Bay Campus, Swansea, SA1 8EN, UK

\author{
Kristian S. Olsen \\ PoreLab, Department of Physics, University of Oslo, Blindern, 0316 Oslo, Norway
}

\begin{abstract}
Silo discharge has been extensively studied for decades although questions remain regarding the nature of the velocity field, particularly for submerged systems. In this work, fluid-driven granular drainage was performed in a quasi-2D silo with grains submerged in fluid. While the observed Gaussian velocity profiles were generally consistent with current diffusion models, the diffusion length was found to significantly decrease with height in contrast to the increases previously seen in dry silos. We propose a new phenomenological anomalous diffusion model for the spreading of the flow upwards in the cell, with the fluid-driven flows we study here falling in the category of subdiffusive behaviour. As the viscous characteristics of the system were amplified, the diffusion length increased and the shape of the flowing zone in the silo changed, deviating further from the parabolic form predicted by traditional normal diffusion models, in effect becoming more subdiffusive as quantified by a decreasing diffusion exponent.
\end{abstract}

\section{INTRODUCTION}

Flow of granular matter in silos is of much industrial interest and has therefore been extensively studied. For the gravitational drainage of dry silos the mass flow rate of grains through an orifice is typically described by the empirical law proposed by Beverloo [1]. It chiefly depends upon the size of the outlet and the size and physical characteristics of the grains and is still the subject of new research [2-7]. Conversely in silos submerged in fluid the flow rate of grains has been seen to differ somewhat from Beverloo's law, exhibiting a considerable surge as the silo is emptied. Only at very high filling heights does the flow rate become constant, whereupon it may be described with a Beverloo-like expression. When the total flow rate is controlled and kept constant however, no such surge is observed and the grain flow rate is steady $[5,8,9]$. Dry silo flows may also be considered multiphase systems with the injection of gas seen to influence grain flow rate [10] and drag from interstitial gas causing strong deviations from Beverloo's law for small grains in closed silos [11].

The flow of grains as they fall inside dry silos has also been widely studied and theorised with various approaches. For decades, the vertical grain velocity in such flow has been observed to be Gaussian in shape [12-14] (see Figure 1). The Gaussian peak is at its largest closest to the outlet, where its standard deviation is smallest. With increasing height these profiles "spread", becoming wider and slower. In this sense the velocity profiles are often thought to be diffusing upwards in the vertical coordinate $y$, rather than in time as with molecular diffusion. As such, the models associated with this flow typically employ the mathematics of diffusion.

One approach to describe this behaviour is the kinematic model proposed by Nedderman and Tüzün [15] which asserts, without explicitly acknowledging a microscopic mechanism, that the horizontal velocity $u$ is pro-

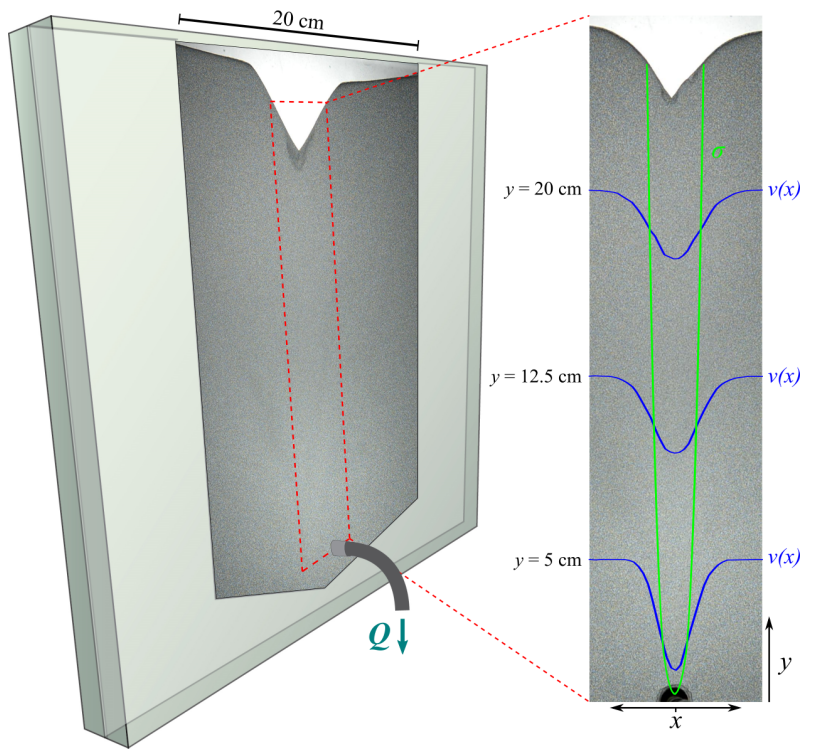

FIG. 1. Left: illustration of the flow cell. Right: Gaussian velocity profiles (blue curves) taken from experiments at various heights $y$ and corresponding standard deviations (green curve) superimposed on the central section of grain flow.

portional to the gradient of the vertical velocity along the horizontal direction $x$ such that $u=-B \frac{\partial v}{\partial x}$ where $B$ is a constant with units of length. Applying mass conservation subsequently results in a diffusion equation and Gaussian vertical velocity profiles are obtained:

$$
v(x)=v_{0} \exp \left(-\frac{x^{2}}{2 \sigma^{2}}\right)
$$

where $\sigma^{2}=2 B y, v_{0}=q_{g} / \sqrt{4 \pi B y}$ with $q_{g}$ being the volumetric grain flux per unit transverse length of the cell. This fits well with experimental observation $[16,17]$; the quantity $B$ has hence been referred to as a "diffusion length", relating to the spreading of the grain velocities 

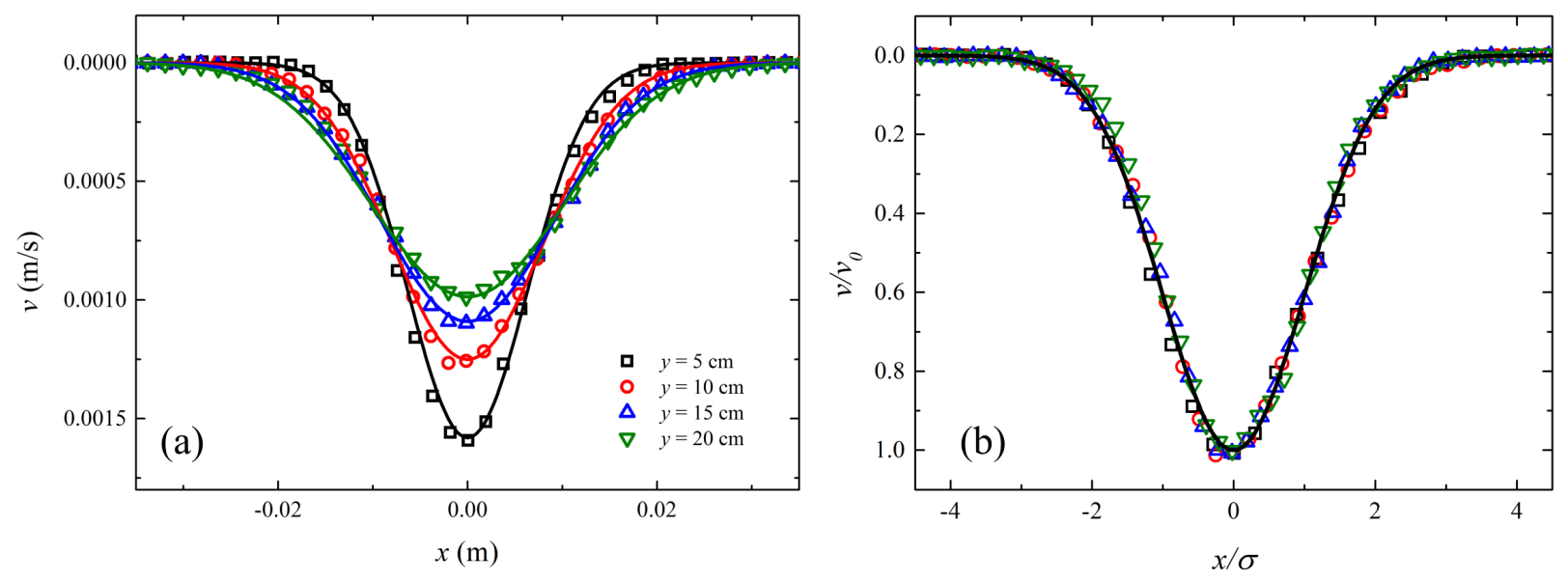

FIG. 2. (a) Vertical velocity profiles at various heights $y$ at $Q=1 \mathrm{ml} / \mathrm{min}$ fitted with Equation 1 . (b) Collapse of Gaussian profiles normalised considering Equation 5 with $\alpha=0.68$ and $B_{\alpha}=1.54 \times 10^{-4} \mathrm{~m}^{(2-\alpha)}$. The black solid line corresponds to the velocity curve in accordance with Equation 5.

vertically up the silo and is typically found to be somewhere between 1.3 to 4 grain diameters $[14,16,18]$.

Several microscopic models have been suggested that can reproduce the kinematic equations, including the void model $[12,13,19]$, where voids originating from the orifice propagate upwards through the cell, and the spot model $[20,21]$ which instead considers some amount of free interstitial volume several grain diameters in size. Spots, like voids, are believed to perform biased random walks, mediating the plasticity of the granular material. The upwards diffusion of spots results in the opposite movement of affected particles and a small change in local volume fraction. The spot model can hence be used to recover Equation 1, whilst employing a more robust microscopic interpretation.

At face value these models consider the diffusion length $B$ a constant and by consequence the flow as adhering to the paradigm of normal diffusion, giving rise to a parabolic flowing zone $\left(\sigma^{2} \sim y\right)$. However, several experimental works have found $B$, obtained from analysis of grain velocity measurements, to increase with height, with the inconsistency between experimental results and theory still unexplained [14-16]. Furthermore, Choi et al. [22] reported that considering a larger $B$ for regions of higher velocity improved fitting with the kinematic model, positing that varying spot density may affect the interaction between different spots and hence their diffusion through the silo. They also found $B$ to be independent of flow rate (orifice size) with velocity profiles coinciding when normalised with flow rate. This is consistent with more recent results in which the orifice size had no clear effect on $B$ [14]. Meanwhile, measurement of grain velocity profiles in submerged silos - both open and flow-controlled - is rare [23] and has not been discussed in reference to the mean velocity models earlier discussed. As such it is not known how applicable the current models are in silos where the flow is fluid-driven with variable flow rates.

This work concerns the fluid-driven flow of grains in a submerged quasi-2D silo. A controlled total volume flow rate of grains and water is withdrawn from an orifice at the front of the silo and vertical velocity profiles are measured. We find that the imposed fluid forces affect not just the rate of discharge, but also the shape of the flowing zone above the outlet - that is to say the growth of Gaussian width $\sigma$ as a function of height up the silo. Fluid forces are increased (relative to gravity) by either increasing the flow rate, increasing the fluid viscosity, or using smaller sized grains. In all cases, the effect is to expand the flowing zone near the outlet, where fluid drag is highest and directed towards the outlet point, such that the shape of the flowing zone becomes more flat near the base. The shape of the flow thus deviates from the parabolic shape of the normal diffusion models, and is instead more accurately described by a sub-diffusive phenomenological model.

\section{METHODOLOGY}

The experimental geometry consisted of a Hele-Shaw cell $30 \mathrm{~cm}$ tall, $20 \mathrm{~cm}$ wide and with an internal gap $b=0.05 \mathrm{~cm}$, illustrated in Figure 1. The cell was filled with water and soda lime glass beads which were allowed to settle, forming a bed of sedimented granular material. Grains were sieved to three size ranges with diameters of between 53 and $100 \mu \mathrm{m}, 100$ to $150 \mu \mathrm{m}$ and 150 to $200 \mu \mathrm{m}$. A total volume flow rate $Q$ was withdrawn with a Harvard PHD Ultra syringe pump from a lateral outlet $4 \mathrm{~mm}$ in diameter at the base and in the centre of one of the plates resulting in the flow of grains and fluid. Withdrawal in this way ensured the grain flow rate was fully controlled by the imposed flow rate with no grain flow occurring without activation of the pump. A second 

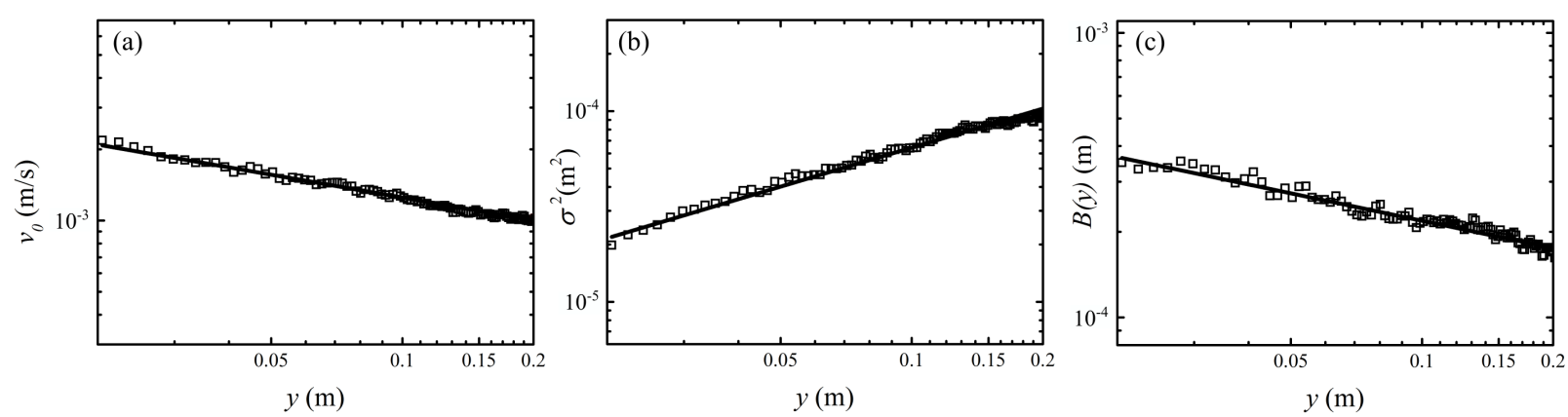

FIG. 3. (a) Peak vertical velocity $v_{0}$ and (b) standard deviation squared $\sigma^{2}$ taken from fits of Equation 1 to vertical grain velocity. Solid lines represent $v_{0}=q_{g} / \sqrt{4 \pi B_{\alpha} y^{\alpha}}$ and $\sigma^{2}=2 B_{\alpha} y^{\alpha}$ with $\alpha$ and $B_{\alpha}$ given in the caption for Figure 2 . (c) $B(y$ ) calculated from data according to $B(y)=\frac{\alpha}{4 \pi y}\left(\frac{q_{g}}{v_{0}}\right)^{2}$ and fitted with Equation 4 .

pump was used simultaneously at the top of the cell to continuously replenish the drained contents with fluid to ensure a constant hydraulic head during the experiment. Additional experiments were carried out using water and glycerol (Sigma Aldrich) mixtures of varying concentration as the interstitial fluid. The cell was backlit with an LED panel and grain motion was captured with a Nikon D7000 camera. Particle image velocimetry (PIV) was subsequently carried out using the PIVlab toolbox in Matlab [24]. Total grain flow rates were measured by integrating PIV vertical velocity profiles and also independently measured by grain depletion from the cell. The consistency between PIV and overall measured flow rates verifies that there are no significant out-of-plane granular velocity gradients and that PIV measurements at the face of the silo are representative for the flow across the narrow gap in the cell. For the PIV measurements, only areas significantly far from the free surface of the bed were considered to avoid edge effects associated with the depression of the free surface that grows over time as the cell is drained.

\section{RESULTS AND DISCUSSION}

\section{A. Velocity field}

Upon initiation of the pump grains flowed downwards and towards the centre in a manner qualitatively similar to behaviour in dry silos, with velocities increasing closer to the outlet. Vertical grain velocities measured by PIV were found to be in good agreement with the Gaussian profiles of Equation 1. Velocity profiles for flow at $Q=1 \mathrm{ml} / \mathrm{min}$ at various heights $y$ are plotted in Figure 2(a) and are fitted with Equation 1 where the Gaussian peak $v_{0}$ and standard deviation $\sigma$ are free parameters. These fitting parameters are plotted as a function of height $y$ in Figure 3. If we consider a constant diffusion length $B$, Equation 1 predicts a linear relationship between $\sigma^{2}$ and $y$ and that $v_{0} \propto y^{-1 / 2}$, however this is not observed under any conditions in this experi- ment. In fact the behaviour may be more appropriately described considering power law relationships $\sigma^{2} \propto y^{\alpha}$ and $v_{0} \propto y^{-\alpha / 2}$, where $\alpha<1$. Within the analogy of diffusion such a relationship, and the variable $B$ with $y$ observed in other work, could be interpreted as being consistent with anomalous behaviour - with $\alpha<1$ indicative of subdiffusion - although this has not before been considered in this way in a steady state silo system. Incidentally, anomalous behaviour has been reported in a different sense regarding particle dynamics during a transient phase at the start of silo discharge $[18,25]$. We can introduce an anomalous characteristic to the spot model by considering the spot diffusivity in power law form $D_{s}(y)=\alpha D_{0}(\alpha) y^{\alpha-1}$. The equation for the spot density $\rho(x, y, t)$ follows the drift-diffusion equation [26]

$$
\frac{\partial \rho_{s}}{\partial t}+v_{s} \frac{\partial \rho_{s}}{\partial y}=\alpha D_{0}(\alpha) y^{\alpha-1} \frac{\partial^{2} \rho_{s}}{\partial x^{2}}
$$

In the steady state the equation simplifies to

$$
\frac{\partial \rho_{s}}{\partial y}=\alpha B_{\alpha}(\alpha) y^{\alpha-1} \frac{\partial^{2} \rho_{s}}{\partial x^{2}}
$$

for which we have introduced $B_{\alpha}=D_{0}(\alpha) / v_{s}$ which has units of length to a power $(2-\alpha)$. The term $\alpha B_{\alpha}(\alpha) y^{\alpha-1}$ may be considered as a $y$-dependent diffusion length mirroring the spot diffusivity

$$
B(y)=\alpha B_{\alpha} y^{\alpha-1} .
$$

Equation 3 has a Gaussian solution, which when normalised to unity takes the form $[27,28]$

$$
\rho_{s}(x, y)=\frac{1}{\sqrt{4 \pi B_{\alpha} y^{\alpha}}} \exp \left(-\frac{x^{2}}{4 B_{\alpha} y^{\alpha}}\right) .
$$

The vertical velocity profile of grains can then, in the simplest case, be written $v(x, y)=q_{g} \rho_{s}(x, y)$ since the motion of the grains takes place where the granular medium has dilated. The proportionality factor $q_{g}$ is the horizontally integrated velocity profile. Hence the velocity Gaussian amplitude and variance are now given by 

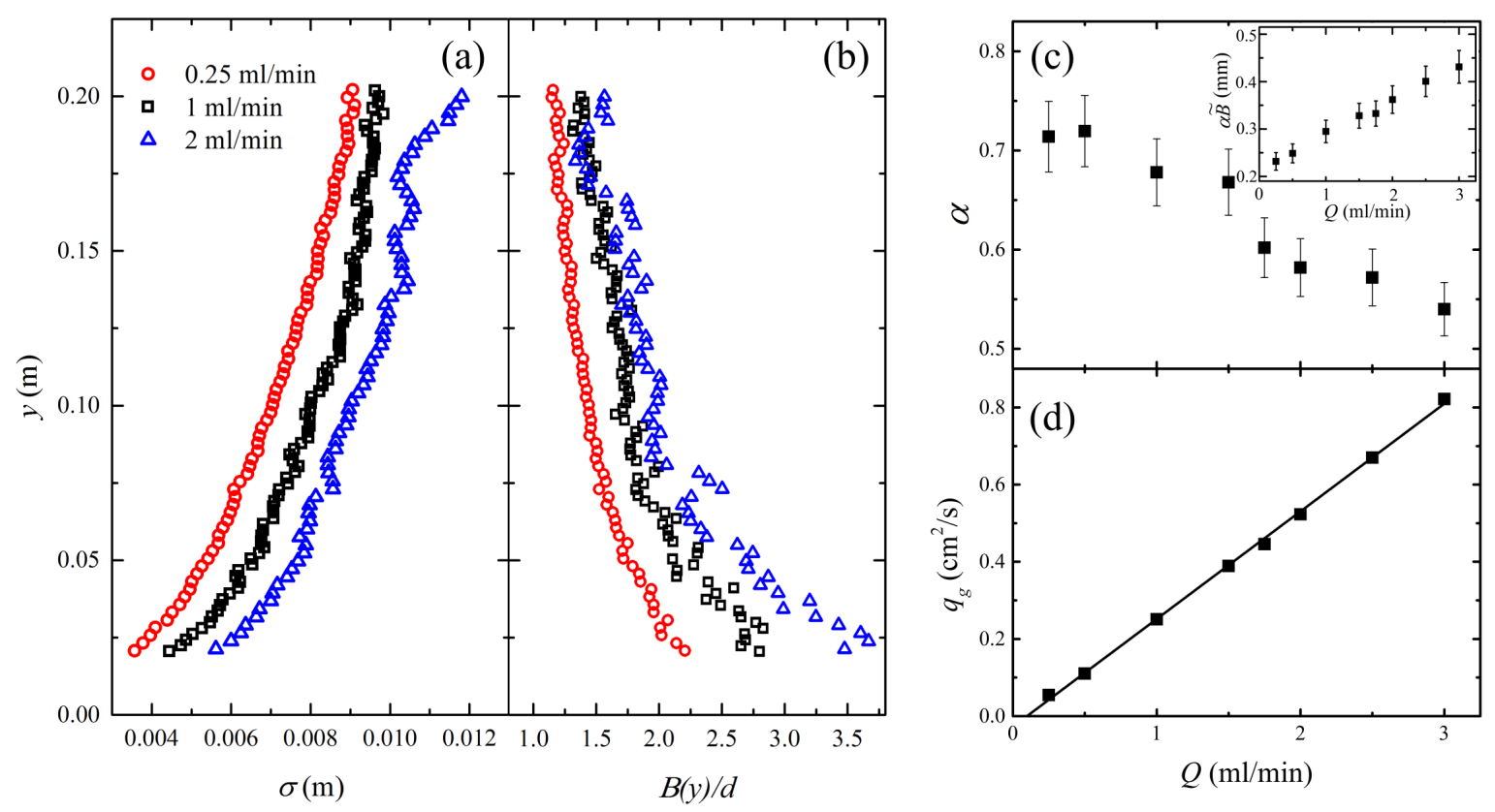

FIG. 4. (a) Standard deviation $\sigma$ and (b) diffusion length $B(y) / d$ calculated as in Figure 3 as a function of height $y$ for a selection of flow rates $Q$. The axes are reversed from Figure 3 and linear to coincide with the silo orientation. (c) The decrease of exponent $\alpha$ as a function of flow rate. Inset: Rescaled diffusion parameter $\alpha \tilde{B}$ indicating the general increase in $B(y)$ as a function of $Q$. (d) Linear increase in grain flux $q_{g}$ with flow rate.

$v_{0}=q_{g} / \sqrt{4 \pi B_{\alpha} y^{\alpha}}$ and $\sigma^{2}=2 B_{\alpha} y^{\alpha}$. These expressions are plotted with data at $Q=1 \mathrm{ml} / \mathrm{min}$ in Figures 3 (a) and (b) where $\alpha=0.68$ and $B_{\alpha}=1.54 \times 10^{-4} \mathrm{~m}^{(2-\alpha)}$ which were obtained from fits of $v_{0}$. Consequently, normalisation of velocity data with respect to $\sigma$ and $v_{0}$ as described by anomalous diffusion provides a collapse as displayed in Figure 2(b) that would not be achieved by considering Equation 1 with a constant $B$. A plot of $B(y)$, obtained from velocity data according to $B(y)=\frac{\alpha}{4 \pi y}\left(\frac{q_{g}}{v_{0}}\right)^{2}$, is displayed in Figure 3(c), fitted with Equation 4, demonstrating its decrease with height up the silo as it approximately halves over the range measured. This is a significant departure from both normal diffusion models where $B$ is a constant, and some observations of increasing $B$ with height in dry, gravity-driven silo flows $[14-16,22]$ which in this context could be consistent with the mathematics of superdiffusion.

\section{B. Flow rate dependence}

The second part of this experiment concerns how the variation of flow rate affects the shape of the flow and the parameters of the phenomenological anomalous diffusion model used to describe it. A range of withdrawal flow rates $Q$ were imposed using the syringe pump at the silo outlet, enabling effective control over grain flow rate. With increasing imposed total flow rate $Q$, the flow rate of grains withdrawn from the outlet was observed to increase linearly once an initial zero flow threshold was surpassed. This is consistent with our previous findings $[29,30]$ and by extrapolation to zero granular flow, this threshold is found to be approximately $0.1 \mathrm{ml} / \mathrm{min}$ in the experiments reported here. This behaviour is displayed in Figure 4(d) where the grain flux $q_{g}$, measured by averaging the numerical integral of $v(x)$ at each height, is plotted as a function of total flow rate.

As with the flow earlier discussed at $Q=1 \mathrm{ml} / \mathrm{min}$, Gaussian velocity profiles that spread outwards with increasing height were observed at all flow rates. To illustrate this, the standard deviation $\sigma$ is plotted with height for various flow rates in Figure 4(a). Interestingly, $\sigma$ becomes larger at higher flow rates; this is different from behaviour observed in dry flows, for which the velocity magnitude merely scales with flow rate [22]. Hence, presently both the Gaussian magnitude and its width increase with flow rate. Meanwhile, at no observed flow rates can the behaviour be adequately modelled using a constant diffusion length; a better description is achieved by considering $B(y)$ according to Equation 4 . The exponent $\alpha$, taken from fits of $v_{0}$ is plotted in Figure 4(c) as a function of $Q$. Even at the lowest flow rates, $\alpha$ is significantly less than 1, indicating anomalous behaviour. As the imposed flow rate is increased the exponent decreases further to $\alpha \approx 0.54$ at $3 \mathrm{ml} / \mathrm{min}$. This is a reflection of the fact that the flowing zone above the outlet is changing shape, becoming wider and flatter near the base with increasing flow rate. This dynamic is captured by the variable diffusion exponent $\alpha$ in this new model. $B(y)$ normalised with grain size $d$ (taken here to be $125 \mu \mathrm{m}$ ) 
is plotted in Figure 4(b) as a function of height $y$ up the silo for selected flow rates. The absolute values of $B(y)$ incidentally increase with $Q$ at a given height, showing that the tendency for the flowing zone to spread outwards is higher (at all cell heights) with increasing flow rate. We can go some way to quantify this by rescaling Equation 4 such that $B(y)=\alpha \tilde{B}\left(\frac{y}{y^{\prime}}\right)^{\alpha-1}$ where the product $\alpha \tilde{B}$ is equivalent to the value of $B(y)$ at a height $y^{\prime}$; for this work we subsequently consider a height of $y^{\prime}=4 \mathrm{~cm}$ which is 10 times the outlet diameter. $\alpha \tilde{B}$ is plotted inset in Figure 4(c), where its increase with flow rate is apparent. Concurrently, the rate at which $B(y)$ decreases with $y$, characterised by $\alpha$, becomes more pronounced. Despite this, the range of $B(y)$ encountered is consistent with that observed in dry flows and it is possible that for a sufficiently tall silo a minimum value would be obtained such that $B(y)>d$. In other words, the effect of fluid forces on the spreading of the flowing zone diminishes further away from the outlet, eventually converging towards a diffusion length on the order of the grain size.

\section{Grain size and fluid viscosity dependence}

To further examine the role of fluid forces on the flow as described by the anomalous diffusion model, the effects of grain size and interstitial fluid viscosity were also considered. Experiments performed with additional grain sizes found qualitatively similar behaviour to that described in the previous section, with $B(y)$ decreasing with height up the silo. Furthermore, the exponent $\alpha$ was found to decrease with $Q$ for all grain sizes, consistent with the results already discussed. The effect of reducing grain size (at a given flow rate) was to produce a flowing zone characterised by lower values of $\alpha$ as shown in Figure

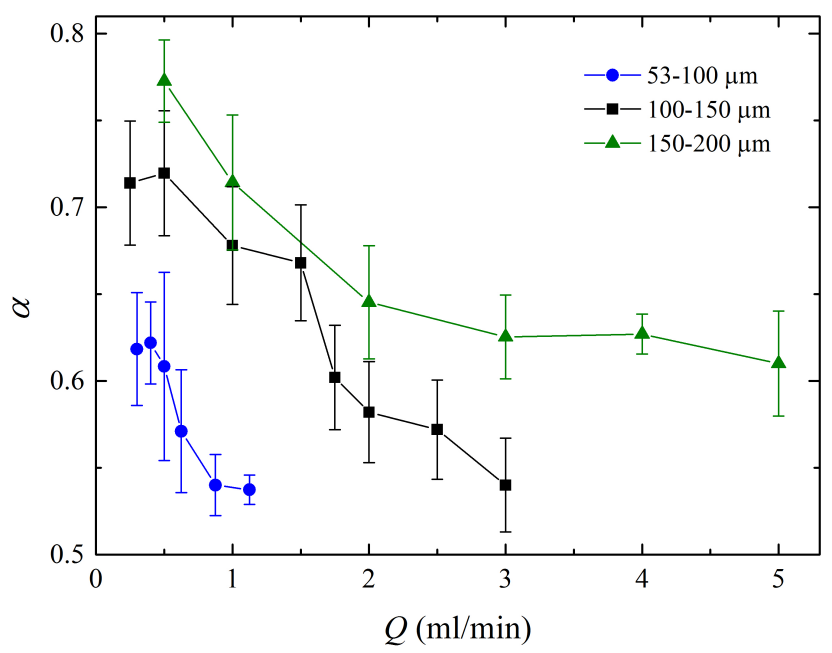

FIG. 5. Exponent $\alpha$ as a function of flow rate for various grain sizes.
5. Hence the smaller the grains, the flatter the shape of the flow at the base of the silo. As with increasing flow rate, Figure 5 therefore shows that decreasing grain size results in flow that deviates further and further from the parabolic upward spreading suggested by the traditional normal diffusion model. (Note that unstable flow develops at lower flow rates for the smaller grains, limiting the available range of $Q$ in the experiments). Meanwhile $\alpha \tilde{B}$, increasing here with $Q$ as before, was found generally to become larger with reduced grain size, signifying a wider flowing zone.

Using aqueous glycerol mixtures between 0 and $40 \mathrm{wt} \%$ the viscosity of the interstitial fluid was varied [31]. A small change in grain buoyancy is acknowledged and considered in later analysis. Tests were carried out at $1 \mathrm{ml} / \mathrm{min}$ with 150 to $200 \mu \mathrm{m}$ grains and showed that with increased viscosity $\alpha$ decreased, as shown in Figure 6 , while $\alpha \tilde{B}$ increased. As such, regarding the anomalous diffusion model of the flow, both decreasing grain size and increasing fluid viscosity appear to have a qualitatively similar effect to that of increasing the flow rate, resulting in generally larger diffusion lengths that decrease more rapidly with distance from the outlet: the flowing zones get wider, and the shape evolves to become flatter at the base. This tendency shares some resemblance with air streamlines through granular media in a gas-injected silo [10] which suggests drag from interstitial fluid flow could influence granular flow in this fashion.

\section{Gravitational and viscous effects}

While the exact physical origin of this behaviour is not currently known, we may speculate with regard to the

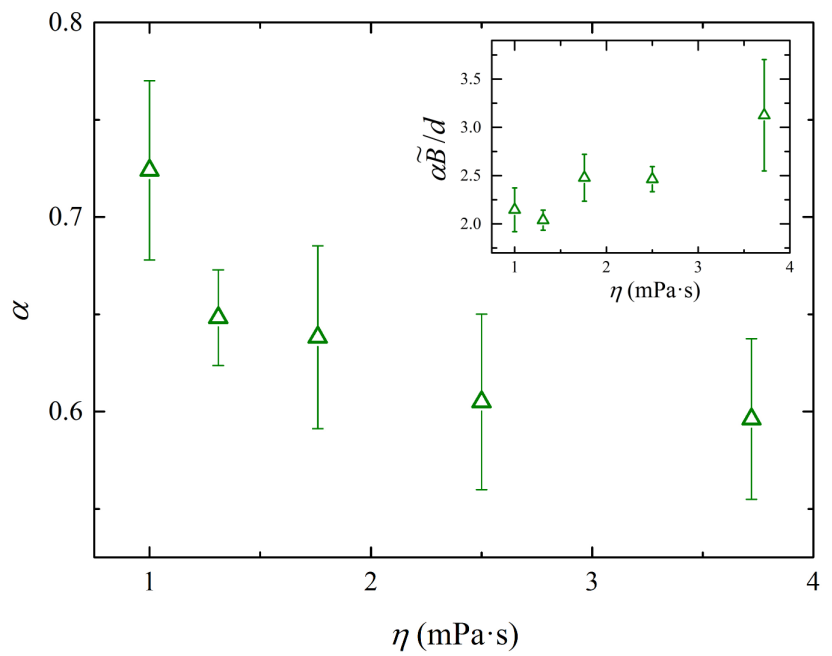

FIG. 6. Exponent $\alpha$ as a function of interstitial fluid viscosity $\eta$ for grains of diameter 150 to $200 \mu \mathrm{m}$ at $1 \mathrm{ml} / \mathrm{min}$. Inset: diffusion length magnitude normalised by grain size $\alpha \tilde{B} / d$ as a function of $\eta$. 


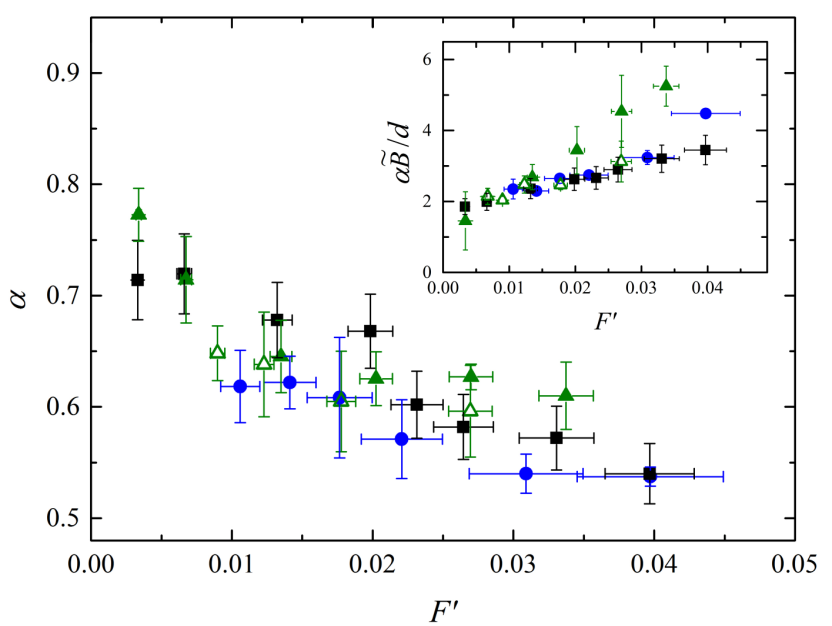

FIG. 7. Exponent $\alpha$ as a function of the dimensionless quantity $F^{\prime}$. Inset: diffusion length magnitude normalised by grain size $\alpha \tilde{B} / d$ as a function of $F^{\prime}$. The legend is as given in Figures 5 and 6 .

mechanisms that affect it. The exaggeration of the apparent subdiffusive behaviour observed in this work coincides with a parametric shift from gravitationally-driven silo discharge, towards a more viscous, flow-induced kind. In order to characterise this we seek to quantify aspects of the flow by considering the relative forces acting on grains in the most elementary sense. The force of gravity acting on a submerged spherical grain can be given by $F_{g}=\frac{\pi}{6} \rho g d^{3}$, where $\rho$ is the buoyancy-corrected grain density and $g$ is gravitational acceleration. To quantify the general viscous effects for each experiment we take the drag force according to Stokes' law for a stationary grain in a moving fluid $F_{d}=3 \pi d \eta v$, where $v=Q / A$ is the superficial fluid velocity with $A$ the silo cross sectional area. A dimensionless parameter $F^{\prime}=F_{d} / F_{g}$ may then be defined that accommodates all present independent variables and represents the relative contributions of viscous and gravitational forces in each experiment.

$$
F^{\prime}=\frac{18 \eta Q}{\rho g d^{2} A}
$$

This ratio could equivalently be described as that between the settling velocity of a grain in fluid and the superficial velocity in the silo imposed by the controlled flow rate. The grain diameter is taken as before as the midpoint between the sieved size ranges, each with a nominal uncertainty of $\pm 5 \mu \mathrm{m}$. The exponent $\alpha$ is plotted with $F^{\prime}$ in Figure 7 whereby $\alpha$ for all experiments loosely collapse, displaying a reduction as $F^{\prime}$ becomes greater. This illustrates the apparent link between the viscous forces induced by the imposed fluid flow and the deviation described by the anomalous diffusion model. It should be noted however that $F^{\prime}$ is still significantly less than unity for all present conditions, suggesting the role of gravity is still considerable.

$F^{\prime}$ may also be employed to compare the diffusion length magnitude $\alpha \tilde{B} / d$ as shown inset in Figure 7, which here has been normalised by grain size. The data coincides well for all experiments but those at the highest flow rates of the largest grains size, with $\alpha \tilde{B} / d$ increasing linearly. This implies that the flowing zone is becoming wider and that the effective diffusion length increases as the imposed flow becomes more dominant. Extrapolation of this trend to a hypothetical $F^{\prime}=0$ for no imposed flow would provide a diffusion length of between $1 d$ and $2 d$. The behaviour illustrated in Figure 7 highlights the importance of fluid drag in this fluid-driven system; it is not known if such trends would continue with interstitial fluid drag acting against grain flow, which is known to reduce grain flow rate $[5,11]$. A possible cause of the disparity concerning the largest grains at high flow rates may be that the grain diameter is becoming comparable to the cell gap and at high flow rates significant dilation could be occurring, widening the flowing region.

\section{CONCLUSIONS}

In summary, velocity profiles of fluid-driven grains in a quasi-2D submerged silo were observed to be Gaussian, as they are in dry silo flows. However the mathematics of normal diffusion with a constant diffusion length, as often presented in models of silo flow could not adequately describe the vertical variation of the velocity field. Instead, by adopting a phenomenological anomalous diffusion model acknowledging a variable diffusivity, the flow throughout the whole packing height was characterised and collapsed. In the submerged system described here, the diffusion length was consistently found to decrease with height. In the context of the anomalous diffusion model this implies subdiffusive behaviour, with diffusion apparently occurring more effectively further down the silo. As the imposed flow rate in the system was increased the flow became more anomalous: the diffusion length decreased more rapidly with height while its general magnitude increased. To this effect the flowing zone became wider and flatter towards the base of the silo. Similar results were observed by increasing the interstitial fluid viscosity and decreasing the grain size - characteristics that coincide with an increase in viscous effects induced by the imposed flow rate.

Future work could examine this further in relation to microscopic models, two phase models and granular temperature. Furthermore, high resolution velocity measurement of dry and certain other submerged systems could clarify whether the description of the diffusion length presented here is also applicable in other scenarios. Such work may provide insight by further exploring the necessary conditions for sub- and potentially superdiffusive flow which could be linked to the drag effects considered here. 


\section{ACKNOWLEDGEMENTS}

We thank Eirik Flekkøy for his discussions. This work was supported by the Engineering and Physical Sciences
Research Council EPSRC grant EP/S034587/1. KSO acknowledges support from the Research Council of Norway through the Center of Excellence funding scheme, Project No. 262644 (PoreLab).
[1] W. Beverloo, H. Leniger, and J. van de Velde, Chemical Engineering Science 15, 260 (1961).

[2] A. M. Cervantes-Álvarez, S. Hidalgo-Caballero, and F. Pacheco-Vázquez, Physics of Fluids 30, 043302 (2018).

[3] S. M. Rubio-Largo, A. Janda, D. Maza, I. Zuriguel, and R. C. Hidalgo, Physical Review Letters 114, 238002 (2015).

[4] K. To, Y. Yen, Y.-K. Mo, and J.-R. Huang, Physical Review E 100, 012906 (2019).

[5] J. Koivisto and D. J. Durian, Nature Communications 8, 15551 (2017).

[6] D. Gella, D. Maza, and I. Zuriguel, Journal of Fluid Mechanics 925, A24 (2021).

[7] R. Blanco-Rodríguez, R. C. Hidalgo, G. Pérez-Ángel, and D. Maza, Granular Matter 23, 86 (2021).

[8] T. J. Wilson, C. R. Pfeifer, N. Mesyngier, and D. J. Durian, Papers in Physics 6, 1 (2014).

[9] M. A. Madrid and L. A. Pugnaloni, Granular Matter 21, 76 (2019).

[10] Y. Zhou, P.-Y. Lagrée, S. Popinet, P. Ruyer, and P. Aussillous, Physical Review Fluids 4, 124305 (2019).

[11] J. E. Hilton and P. W. Cleary, Physical Review E 84, 011307 (2011).

[12] J. Litwiniszyn, Rheologica Acta 1, 146 (1958).

[13] W. Mullins, Powder Technology 23, 115 (1979).

[14] I. Zuriguel, D. Maza, A. Janda, R. C. Hidalgo, and A. Garcimartín, Granular Matter 21, 47 (2019).

[15] R. Nedderman and U. Tüzün, Powder Technology 22, 243 (1979).

[16] A. Medina, J. Córdova, E. Luna, and C. Treviño, Physics Letters A 250, 111 (1998).
[17] A. Garcimartín, I. Zuriguel, A. Janda, and D. Maza, Physical Review E - Statistical, Nonlinear, and Soft Matter Physics 84, 1 (2011).

[18] J. Choi, A. Kudrolli, R. R. Rosales, and M. Z. Bazant, Physical Review Letters 92, 174301 (2004).

[19] H. Caram and D. C. Hong, Physical Review Letters 67, 828 (1991).

[20] M. Z. Bazant, Mechanics of Materials 38, 717 (2006).

[21] K. Kamrin and M. Z. Bazant, Physical Review E 75, 1 (2007).

[22] J. Choi, A. Kudrolli, and M. Z. Bazant, Journal of Physics: Condensed Matter 17, S2533 (2005).

[23] A. V. Orpe and A. Kudrolli, Physical Review Letters 98, 238001 (2007).

[24] W. Thielicke and E. J. Stamhuis, Journal of Open Research Software 2 (2014).

[25] R. Arévalo, A. Garcimartín, and D. Maza, European Physical Journal E 23, 191 (2007).

[26] H. Risken, The Fokker-Planck Equation (Springer, 1996).

[27] S. C. Lim and S. V. Muniandy, Physical Review E 66, 021114 (2002).

[28] B. I. Henry, T. A. M. Langlands, and P. Straka, in Complex Physical, Biophysical and Econophysical Systems, 1828 (World Scientific, 2010) pp. 37-89.

[29] M. L. Morgan, D. W. James, A. R. Barron, and B. Sandnes, Physics of Fluids 32, 113309 (2020).

[30] M. Morgan and B. Sandnes, EPJ Web of Conferences 249, 03041 (2021).

[31] J. B. Segur and H. E. Oberstar, Industrial \& Engineering Chemistry 43, 2117 (1951). 